\title{
Assessment of inflammatory infiltration and angiogenesis in the thrombus and the wall of abdominal aortic aneurysms on the basis of histological parameters and computed tomography angiography study
}

\author{
Adam Lukasiewicz ${ }^{1}$, Joanna Reszec ${ }^{2}$, Radoslaw Kowalewski ${ }^{3}$, \\ Lech Chyczewski ${ }^{2}$, Urszula Lebkowska ${ }^{1}$ \\ ${ }^{1}$ Department of Radiology, Medical University of Bialystok, Poland \\ ${ }^{2}$ Department of Medical Pathomorphology, Medical University of Bialystok, Poland \\ ${ }^{3}$ Department of Vascular Surgery and Transplantology, Medical University of Bialystok, Poland
}

\begin{abstract}
The proliferation of vessels within the aneurysm's wall and the intraluminal thrombus of abdominal aortic aneurysm (AAA) may be the main factor responsible for progression and rupture of AAA. The aim of this study was to compare the parameters of the thrombus (size, density, contrast enhancement) measured by computed tomography (CT) with histological assessment of thrombi removed during surgery. 29 patients with AAA were examined with angio-CT. Post-surgery histopathological evaluation of AAA was performed. Slides were stained with markers of B- (CD20) and T-lymphocytes (CD3), and markers of endothelial cells (CD34). Thrombi were enhanced after contrast media administration in angio-CT $(p=0.002)$. There was a statistically significant correlation between contrast enhancement and the presence of B lymphocytes. Intensity of endothelial cell marker expression significantly correlated with the presence of inflammatory T- and B-cells. No statistical significant correlation was found between contrast enhancement of the thrombus and markers of endothelial cells. The accumulation of inflammatory cells in the wall of AAA thrombus results in the formation of new vessels which participates to the instability of the thrombus and AAA wall. Assessment of the inflammation and neovascularization in the wall and thrombus of the AAA might be an important factor in monitoring the progression and the risk of aneurysm's rupture. (Folia Histochemica et Cytobiologica 2012, Vol. 50, No. 4, 547-553)
\end{abstract}

Key words: abdominal aortic aneurysm, thrombus, CD3, CD20, CD34, angiogenesis, CT angiography.

\section{Introduction}

The research on processes occurring in the wall and in the intraluminal thrombus of abdominal aortic aneurysm proves that the disease-affected wall and in-

Correspondence address: A. Lukasiewicz,

Department of Radiology,

Medical University of Bialystok,

M. Sklodowskiej-Curie St. 24a, 15-276 Bialystok, Poland; tel.: + 488574682 18;

e-mail: adador@tlen.pl traluminal thrombus development are the centre of an intense angiogenesis process [1-5].

The proliferation of small vessels within the aneurysm wall and in the intraluminal thrombus of abdominal aortic aneurysm (AAA) may be one of the factors responsible for progression of the AAA and the increased risk of rupture [6,7]. It has been proved that the course of AAA is connected with neovascularization, inflammatory processes activity, free oxygen radicals activity and also with the width of the mural thrombus [8]. Mayranpaa et al. and Kazi et al. revealed the close link between intraluminal throm- 
bus, inflammation and neovascularization within aneurysmal wall, that may decrease integrity and stability of the aneurysm wall leading to its rupture $[9,10]$.

The quantitative evaluation of neovascularization in the AAA wall is possible only in the histopathological examination of the preserved tissues sample after AAA surgery [11].

Modern imaging methods, despite the fact of their continual improvement, allow only morphologic and qualitative thrombus assessment.

The aim of this study was to estimate the morphological parameters of the AAA thrombus (size, density, contrast enhancement) obtained by computed tomography angiography (CTA) and correlation with special histological parameters of thrombus removed during surgery.

\section{Material and methods}

29 patients with AAA (27 males, 2 female; aged 56-80, average age 70) were examined with CTA and in the next stage, after surgery, histopathological examination was done, including estimation of the inflammation and new vessels proliferation.

All CT examinations were obtained with CT scanner Aquilion 16 (Toshiba Co., Ltd., Tokyo, Japan) equipped with 16 detectors with the following scanning parameters: a gantry rotation speed of $0.4 \mathrm{sec}$ per rotation, detector row configuration of $16 \times 1 \mathrm{~mm}$, helical pitch 23 (pitch factor $1,4375)$. Images were reconstructed with a $1-\mathrm{mm}$ slice thickness at $0,8 \mathrm{~mm}$ intervals. Contrast agent (Iomeron 400, Nycomed) was administered by infusion pump through an antecubital vein to enhance the aorta and iliac arteries followed by saline infusion flush. Contrast volume was estimated according to the rule (time of scanning $+10 \times 4$ ) and infusion rate of $4 \mathrm{ml} / \mathrm{s}$ was set. Coordination between contrast administration and image acquisition was adjusted automatically by the scanner (SUREStart) when $180 \mathrm{HU}$ of contrast concentration was detected at a region of interest located in descending aorta. Axial images were analyzed on available workstation (Vitrea 4.2, Vital Inc.)

The thrombus density measurements were done by drawing circle or elliptical regions of interest (ROI) in the axial CT scans at several different levels including maximal diameter of the AAA sac and level of maximal thrombus width. The ROIs at each level were set at three localization: close to the lumen, near the wall of aneurysm and in the middle of thrombus. Average densities of ROIs from pre- and post-contrast phase were estimated and then compared (Figure 1).

For the histopathological evaluation the specimen fragments of aorta wall and the thrombus were taken during surgery and were fixed in $10 \%$ buffered formalin. Evaluation of inflammatory cells markers expression was per-

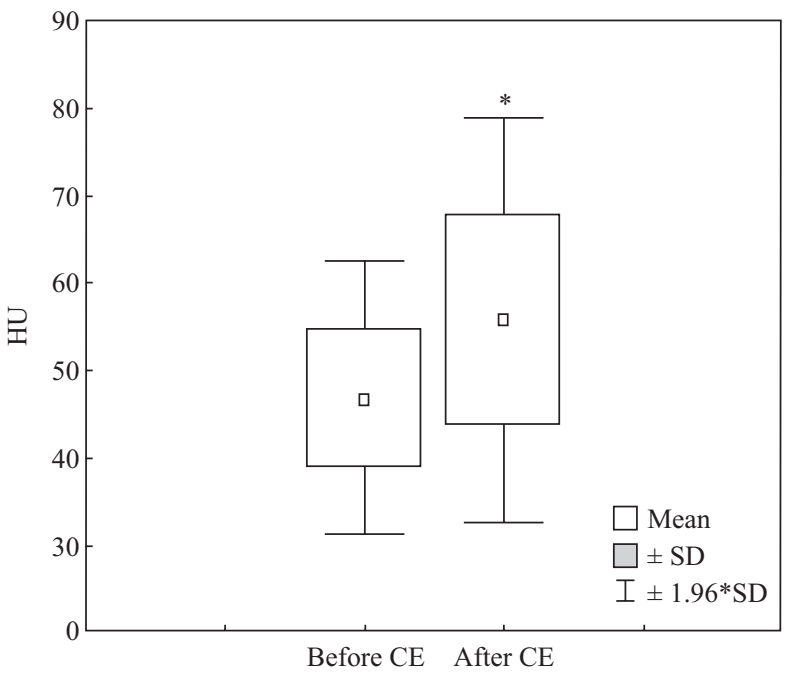

Figure 1. AAA wall density in Hounsfield units (HU) based on the CT analysis of thrombi, before and after administration of the contrast medium (CE, contrast enhancement). $* \mathrm{p}=0.002(\mathrm{n}=29)$

formed using immunohistochemical method. Following the deparaffinision and rehydration, epitope retrieval was carried out in the EnVision Flex Target Retrieval Solution (DAKO) in high $\mathrm{pH}$. Endogenous peroxidases were blocked by incubating the sections in methanol and $30 \%$ hydrogen peroxydase for 20 minutes. Then slides were incubated with special types of antibodies against following antigens 12): CD3, a common antigen for all types of T lymphocytes cells; CD20, a common receptor of B lymphocytes; CD34, a common endothelial cells marker was used to count a number of new proliferating vessels in the thrombus. CD34 visualisation together with HE examination enabled us to count only small new blood vessels in the thrombus. Monoclonal mouse antibody against CD20 receptor was used (M0755, DAKO) in 1:200 dilution overnight in $4^{\circ} \mathrm{C}$ and polyclonal rabbit antibody against $\mathrm{CD} 3$ receptor (A0452, DAKO) in 1:100 dilution overnight in $4^{\circ} \mathrm{C}$. To evaluate new vessels mouse antibody against CD34 receptor was used (IR632, DAKO) in 1:100 dilution incubated overnight in $4^{\circ} \mathrm{C}$. Visualisation reagent (LSAB plus, DAKO) was applied for 30 minutes followed by DAB solution for 5 minutes. The slides were than counterstained with hematoxylin and examined under the light microscope Olympus BX45. Immunohistochemical evaluation of antigens' expression was performed by two independent pathologists. The reactions for CD3 and CD20 were estimated as the percentage of positively-stained cells as follows: $\leq 10 \%$ positive cells - negative $(-), 11-$ $-30 \%(+), 31-50 \%(++)$ and $>50 \%$ as $(+++)$ of positive cells in random 10 fields under $20 \mathrm{x}$ magnification. To measure the numbers of new vessels we counted all of the CD34 positive areas in random 10 fields under $10 \mathrm{x}$ magnification. The score was negative (-), if there was no CD34 positive 


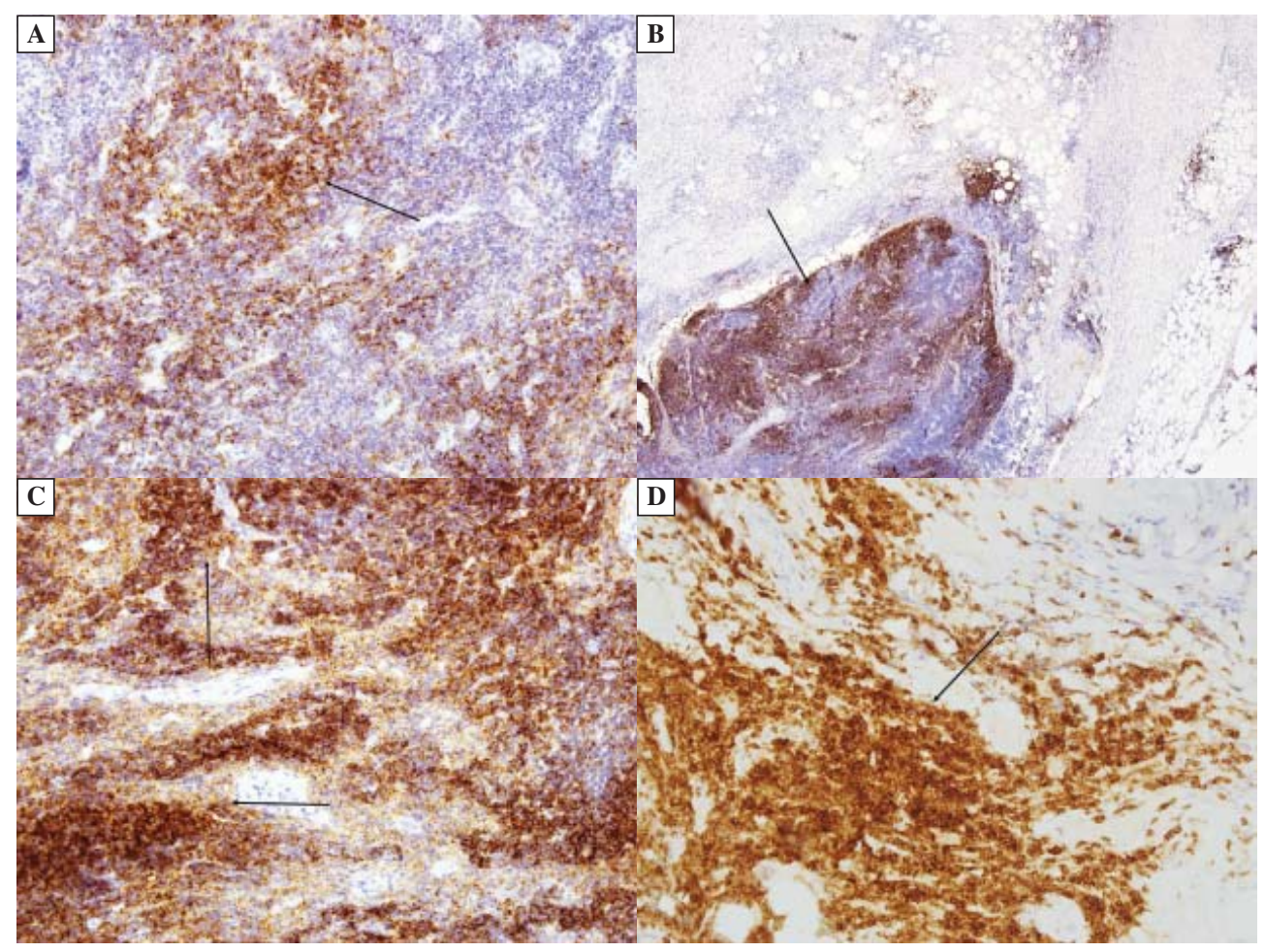

Figure 2A and B. CD3 expression in inflammatory cells (T-cells) within the thrombus. Magnification $200 \times$, and $100 \times$, respectively. C and D. CD20 expression in inflammatory cells (B-cells) within the thrombus, $200 \times$, and $100 \times$, respectively

cells in all of the examined areas, $(+)$ if less than $10 \%$ of the examined fields were CD34 positive; $(++)$ if between $11-50 \%$; and $>50 \%$ as $(+++)$ of the examined fields were CD34 positive.

Negative controls were performed using a nonimmunized IgG replacing the primary antibody. Positive controls involved sections of normal lymph node which expressed CD3 and CD20 antigens and angiosarcoma which expressed CD34 molecules.

$\chi^{2}$ and Pearson's correlation tests and Statistica 8,0 StatSoft software were used for statistical analysis. Values of $\mathrm{p}<0,05$ were considered as statistically significant.

\section{Results}

Microscopic evaluation of the thrombi showed huge percentage of inflammatory cells positive for CD3 or CD20 staining (Figures 2A-D) and new vessels proliferation (Figures 3A-D) in the wall, but also in the thrombus of abdominal aortic aneurysms. CD3, CD20 and CD34 expression were found respectively in $14(48 \%), 10(34 \%), 9(31 \%)$ cases of thrombus specimens and in $22(76 \%), 19(65,5 \%), 19(65,5 \%)$ fragments of aortic wall specimens (Table 1).

Significant numbers of inflammatory cells and new vessels were observed both in the wall and the thrombus of abdominal aortic aneurysms (Figures 2 C-D,
3 B-D), which in CT have had morphological signs of inflammatory process surrounding the AAA (visible infiltrations outside the wall of AAA in CT examination).

In CT angiography thrombi were slightly (average 3,5-6,9 HU), but statistically significantly, enhanced after contrast media administration (Figure 1).

There was also a statistically significant correlation between the presence of infiltrating B-cells and contrast enhancement in CT (Figure 4), although we did not observed significant correlations between contrast enhancement of thrombi with the presence of T-cells $(r=0,32, p=0,09)$ and endothelial cells $(\mathrm{r}=0,32 ; \mathrm{p}=0,086)$.

The number of new blood vessels assessed by CD34 expression correlated with the presence of inflammatory cells, both $\mathrm{T}$ and $\mathrm{B}$ lymphocytes (Figure 5).

Negative correlation was observed between contrast enhancement in CT and the maximum diameter of the aneurysm and width of the thrombus (Figures 6A, B).

\section{Discussion}

Multi-factor etiology of AAA and the latent course of the disease are frequently the causes of the systematical increase in the aorta diameter lasting for years, undiagnosed clinically yet $[6,13,14]$. Frequent- 


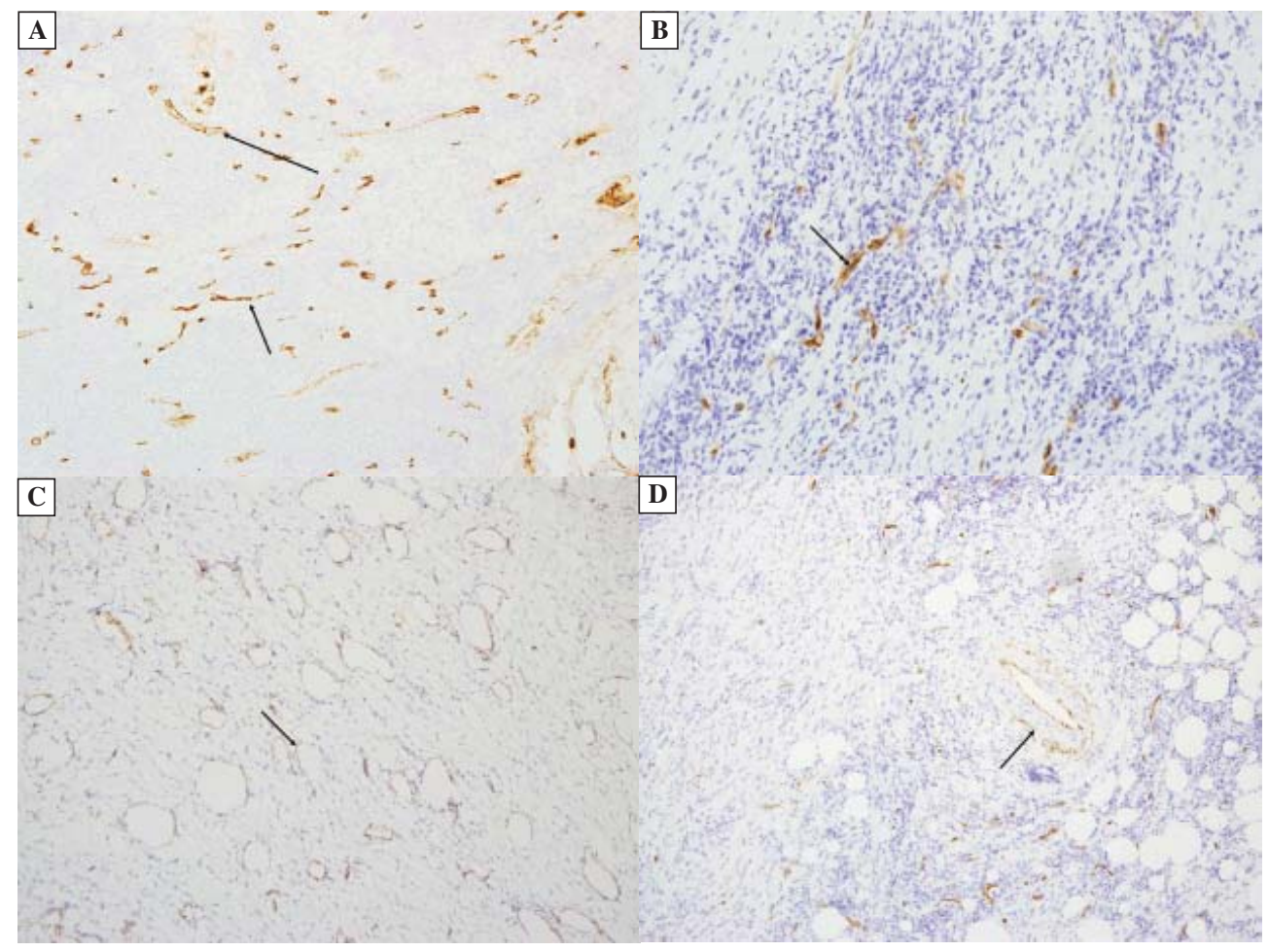

Figure 3. CD34 expression in endothelial cells (arrows). A. New small blood vessels within the wall of the AAA thrombus, $100 \times$. B. Few small blood vessels surrounded by huge inflammatory cells infiltration within the AAA thrombus, $200 \times$. C and D. Matured blood vessels in the AAA thrombus, $200 \times$ and $100 \times$, respectively

Table 1. Expression of cell markers of CD3 (T lymphocytes), CD20 (B lymphocytes), CD 34 (endothelial cells) within the thrombus and the wall of abdominal aortic aneurysm

\begin{tabular}{|l|cc|cc|cc|}
\hline \multirow{2}{*}{$\begin{array}{l}\text { Intensity of } \\
\text { expression* }\end{array}$} & \multicolumn{2}{|c|}{ CD3 } & \multicolumn{2}{c|}{ CD20 } & \multicolumn{2}{c|}{ CD34 } \\
\cline { 2 - 8 } & Thrombus & Wall & \multicolumn{2}{c|}{ Thrombus Wall } & \multicolumn{2}{c|}{ Thrombus Wall } \\
\hline$(-)$ & 15 & 7 & 19 & 10 & 20 & 10 \\
\hline$(+)$ & 7 & 10 & 7 & 15 & 2 & 7 \\
\hline$(++)$ & $\mathbf{2}$ & $\mathbf{5}$ & $\mathbf{2}$ & $\mathbf{2}$ & $\mathbf{2}$ & $\mathbf{6}$ \\
\hline$(+++)$ & $\mathbf{5}$ & $\mathbf{7}$ & $\mathbf{1}$ & $\mathbf{2}$ & $\mathbf{5}$ & $\mathbf{6}$ \\
\hline
\end{tabular}

Numbers in the table represent number of patients in a given group $\left(-,+,{ }_{-}++,+++\right)$. *expression of CD3, CD20, and CD34 markers was estimated as described in material and methods

ly, the diagnosis is made as soon as the initial symptoms of the aneurysm rupture will appear [15]. However, the patient is then given little chance of a successful operation, because acute aneurysm rupture results in death up to $90 \%$ of cases, regardless of the urgent surgery procedures in such cases.

Nowadays AAA diagnosis is made basing on abdominal ultrasonography examination, often done for other reasons, for instance the growth spread of AAA might be systemically controlled, if is below $5 \mathrm{~cm}$ in diameter through about 6 moths [15].

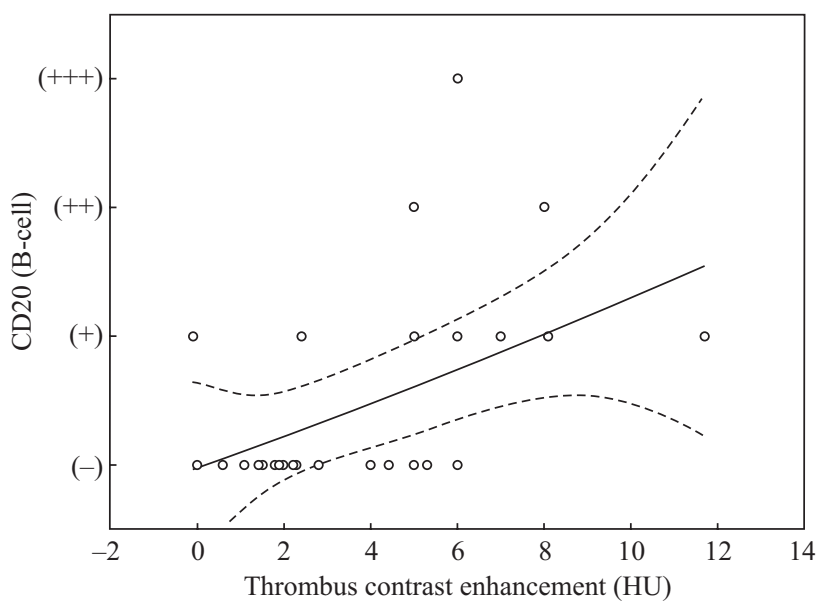

Figure 4. Correlation between contrast enhancement (HU, Hounsfield unit) with B-cell infiltration $(r=0.5, p=0.005)$ within aneurysmal thrombus. $Y$ axis: numbers of $C D 20$ positive cells in random 10 fields at the magnification $20 \times$ are denoted as described in material and methods

Randomized studies have shown that the surgery of small AAA (below $5.5 \mathrm{~cm}$ in diameter) poses higher death risks compared to monitoring of the disease course. In such case "watchful waiting", which is a systematical surveillance, yields better results as far 


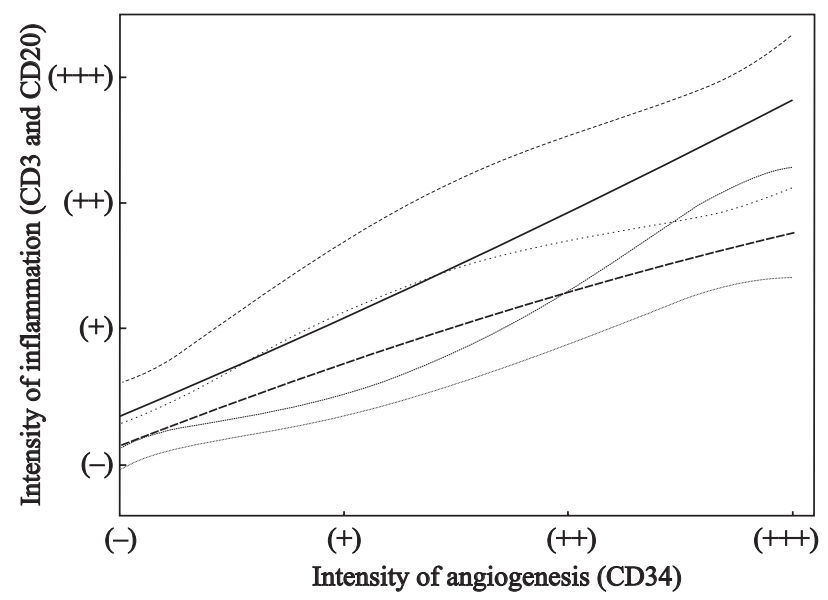

Figure 5. Correlation between expression of CD34, endothelial cell marker, and the presence of inflammatory T cells $(r=0,608, p=0,027)$ and $B$ cells $(r=0,727, p=$ $0,005)$. Y axis: numbers of CD34 cells in random 10 fields at the magnification $20 \mathrm{x}$ are denoted as described in material and methods

as the long-term survival is concerned [16]. Nevertheless, the diameter above $5.5 \mathrm{~cm}$ results in immediate-scheduling for operation on AAA.

The current attempts in the diagnostics path of AAA are to better predict AAA progression to prevent rupture. The basic etiologic factor of the disease is well known: decrease durability of the vessel wall with age due to the degradation of elastin fibers and the transformation of their spatial structure, which results in the vessel widening [17-20].

Damage of the vessel wall and endothelium shortly activate coagulation and thrombosis. Recently, it is admitted that such thrombus may be the main factor leading to further weakening of the vessel wall, disease progression, and in consequence AAA rupture $[21,22]$. The growing thrombus impairs the wall nourishing, causing decreased oxygenetion and ill-distribution of nutrients from blood to the vessel's wall. The local hypoxia activates neutrophils and lymphocytes chemotaxis and stimulates angiogenesis by activating growth factors, like Vascular Endothelial Growth Factor (VEGF) [23, 24].

It was discovered, that inflammation and new vessels proliferation within the wall of abdominal aortic aneurysms and within the thrombus might be confirmed by medical imaging technique [23-26]. Our present results show straight correlation between B cells inflammatory infiltration and contrast enhancement in CT. Similarly, Spadaccio et al. observed in AAA subjects four serum inflammatory proteins that show altered expression profile and that could be specifically linked to AAA pathology [27]. Also Houard $\mathrm{X}$ et al. indicated neutrophil-derived leukotriene B4
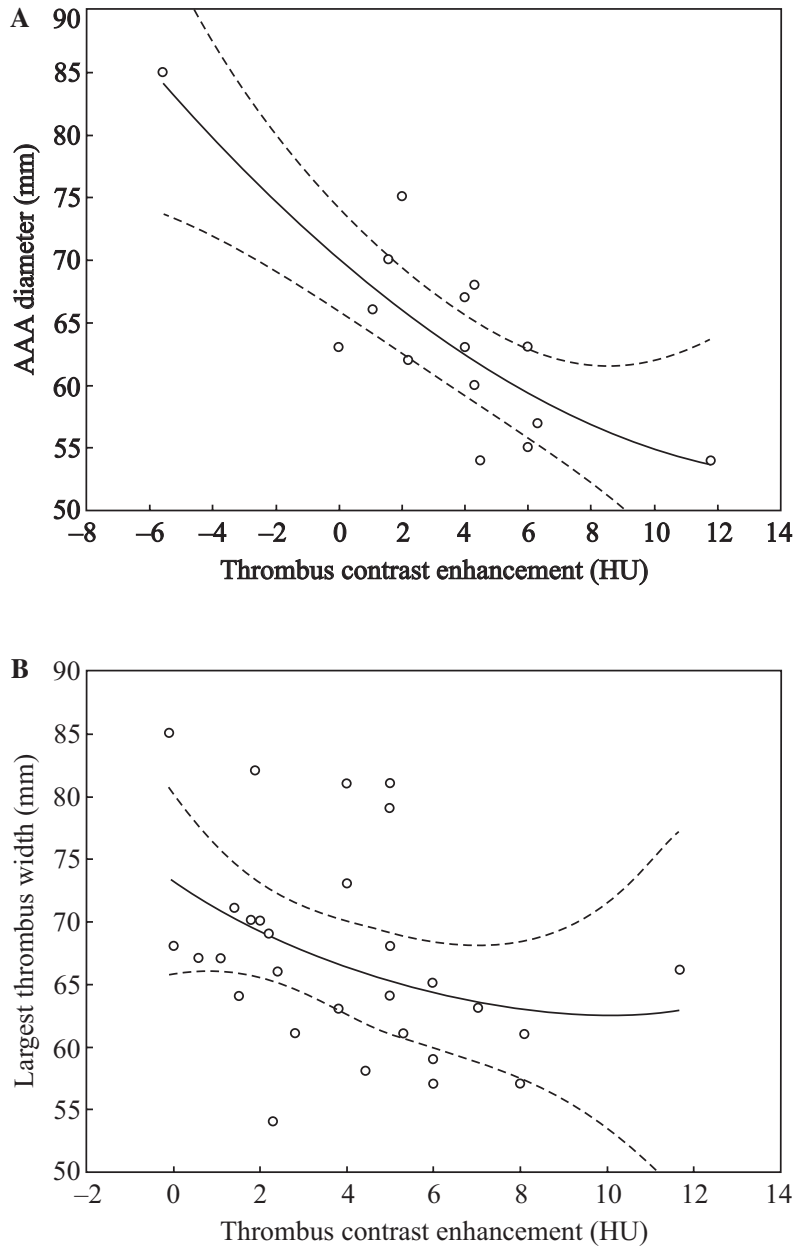

Figure 6. Negative correlation between contrast enhancement and the maximal diameter of the aneurysm (A) and the largest thrombus width $(\mathbf{B}), \mathrm{r}=-0.515, \mathrm{p}=0.004$ and $\mathrm{r}=-0.738, \mathrm{p}=0.002$, respectively

(LTB) as a major neutrophil chemotactic factor released from the intraluminal thrombus of human AAAs, suggesting that targeting LTB receptors may represent a potential therapeutic strategy in the prevention of AAA progression in humans [3]. Kazi et al. suggested that thrombus formation and accumulation of inflammatory cells may perturb the structural integrity and stability of the vessel wall and thereby increase the risk for aneurysm rupture [12]. Also Wolski et al. observed within the AAA thrombus a huge inflammatory cells infiltration together with intensive angiogenesis [28]. Vorp et al. observed localized hypoxia in regions of thicker intraluminal thrombus in AAA, leading to increased, localized mural neovascularization and inflammation, as well as regional wall weakening [29]. They concluded that intraluminal thrombus may play an important role in the pathology and natural history of AAA. Reeps et al. observed that increased 18F-fluorodeoxyglucose 
(FDG) uptake was correlated with higher densities of inflammatory infiltrates with reduced content of collagen fiber, suggesting that FDG-PET/CT might be a new diagnostic technique to study AAA disease in vivo and may contribute to improved prediction of individual AAA rupture risk [30].

Local hypoxia and the presence of inflammatory cells might stimulate the secretion of many growth factors such as VEGF, FGF, TGF, EGF or cytokines like interleukin 8 (II-8), resulting in new vessels growth and, in consequence, in instability within the aortic wall and thrombus [31,32]. Many cytokines and metaloproteinases (MMP-3 or MMP-9), secreted by inflammatory cells, are responsible for further structural proteins like elastin, collagen and laminin degradation of the vessel wall, resulting in a gradual weakening of the wall strength. Therefore, inflammation and angiogenesis may be important events associated with the AAA progression and its rupture [33-35] as was also demonstrated in our present study by showing correlation between CD34 expression and lymphocytic infiltration.

Wiernicki et al. observed increased activity of elastase as well as concentrations of active metaloproteinases like MMP-9, MMP-8 in the thin thrombus-covered wall, demonstrating the differentiation of protease activity within the same AAA wall and its enhancement within the thin thrombus-covered aneurysm wall [36]. Michel et al. observed neoangiogenesis, phagocytosis by mononuclear cells, and a shift from innate to adaptive immunity in the adventitia in the AAA [37] Similar Mayranpaa et al. presented mRNA increased levels of genes associated with neovascularization (VEGF, FLT1, VE-cadherin, CD31), and mast cells (tryptase, chymase, cathepsin $\mathrm{G})$ in AAA samples than in controls [38].

It might be presumed, that the activation of inflammatory process in the thrombus wall is closely related to angiogenesis and may be directly proportional to the risk of AAA rupture. The best way to demonstrate the new vessels proliferation within the thrombus is microscopic assessment of specimens, sampled during operation, whereas clinical imaging methods give images of living tissues, but their spatial resolution is not enough to directly demonstrate new vessels $[39,40]$.

However developing new techniques in modern imaging methods for example new contrast media in ultrasonography (US), magnetic resonance imaging (MRI), computed tomography (CT) and in positron emission tomography (PET) increase the opportunity to use these methods for the evaluation of angiogenesis process. Angio-CT and MRI techniques enable measurements of the contrast enhancement of the thrombus and the AAA wall. Our study presents close link between activation of the inflammatory cells and new vessels proliferation. Inflammatory process may increase thrombus enhancement in CT, what may be an easy way to assess the degree of inflammation and neovascularization used for monitoring the status of AAA and assessing risk of its rupture.

\section{Conclusions}

The accumulation of inflammatory cells in the wall of AAA thrombus results in the formation of new vessels which participates to the instability of the thrombus and AAA wall. Assessment of the inflammation and neovascularization in the wall and thrombus of the AAA might be an important factor in monitoring the progression and the risk of aneurysm's rupture.

\section{References}

1. Thubrikar MJ, Robicsek F, Labrosse M, Chervenkoff V, Fowler BL. Effect of thrombus on abdominal aortic aneurysm wall dilation and stress. J Cardiovasc Surg. (Torino). 2003;44:67-77.

2. Kobayashi M, Matsubara J, Matsushita M, Nishikimi N, Sakurai T, Nimura Y. Expression of angiogenesis and angiogenic factors in human aortic vascular disease. J Surg Res. 2006;106:239-245.

3. Houard X, Ollivier V, Louedec L, Michel JB, Back M. Differential inflammatory activity across human abdominal aortic aneurysms reveals neutrophil-derived leukotriene B4 as a major chemotactic factor released from the intraluminal thrombus. FASEB J. 2009;23:1376-1383.

4. Choke E, Thompson MM, Dawson J et al. Abdominal aortic aneurysm rupture is associated with increased medial neovascularization and overexpression of proangiogenic cytokines. Arterioscler Thromb Vasc Biol. 2006;26:2077-2082.

5. Choke E, Cockerill GW, Dawson J et al. Increased angiogenesis at the site of abdominal aortic aneurysm rupture. Ann N Y Acad Sci. 2006;1085:315-319.

6. Angouras D, Sokolis DP, Dosios T et al. Effect of impaired vasa vasorum flow on the structure and mechanics of the thoracic aorta: implications for the pathogenesis of aortic dissection. Eur J Cardiothorac Surg. 2000;17:468-473.

7. Ballard DJ, Filardo G, Fowkes G, Powell JT. Surgery for small asymptomatic abdominal aortic aneurysms. Cochrane Database Syst Rev. 2008;CD001835.

8. Bluestein D, Niu L, Schoephoerster RT, Dewanjee MK. Steady flow in an aneurysm model: correlation between fluid dynamics and blood platelet deposition. $J$ Biomech Eng. 1996;118:280-286.

9. Byrnes G, Mac Gowan WA. The injury potential of Fogarty balloon catheters. J Cardiovasc Surg. (Torino) 1975;16:590-593.

10. Carmeliet P. Angiogenesis in health and disease. Nat Med. 2003;9:653-660.

11. Buckley DL. Uncertainty in the analysis of tracer kinetics using dynamic contrast-enhanced T1-weighted MRI. Magn Reson Med. 2002;47:601-606.

12. Kazi M, Thyberg J, Religa $P$ et al. Influence of intraluminal thrombus on structural and cellular composition of abdominal aortic aneurysm wall. J Vasc Surg. 2003;38:1283-1292. 
13. Di Martino ES, Guadagni G, Fumero A et al. Fluid-structure interaction within realistic three-dimensional models of the aneurysmatic aorta as a guidance to assess the risk of rupture of the aneurysm. Med Eng Phys. 2001;23:647-655.

14. Pearce WH, Shively VP. Abdominal aortic aneurysm as a complex multifactorial disease: interactions of polymorphisms of inflammatory genes, features of autoimmunity, and current status of MMPs. Ann N Y Acad Sci. 2006;1085:117-132.

15. U.K. Small Aneurysm Trial Participants. Long-term outcomes of immediate repair compared with surveillance of small abdominal aortic aneurysms. N EnglJ Med. 2002;346:1445-1452.

16. Filardo G, Powell JT, Martinez MA, Ballard DJ. Surgery for small asymptomatic abdominal aortic aneurysms. Cochrane Database System Rev. 2012;14:CD001835.

17. Michel JB, Martin-Ventura JL, Egido J et al. Novel aspects of the pathogenesis of aneurysms of the abdominal aorta in humans. Cardiovasc Res. 2011;90:18-27.

18. Powell JT, Brown LC. The natural history of abdominal aortic aneurysms and their risk of rupture. Acta Chir Belg. 2001;101:11-16.

19. Pucci A, Martino S, Celeste A et al. Angiogenesis, tumor necrosis factor-alpha and procoagulant factors in coronary artery giant aneurysm of a fatal infantile Kawasaki disease. Cardiovasc Pathol. 2008;17:186-189.

20. Wang DHJ, Makaroun M, Webster MW, Vorp DA. Mechanical properties and microstructure of intraluminal thrombus from abdominal aortic aneurysm. J Biomech Eng. 2001;123: 536-539.

21. Crowther M, Goodall S, Jones JL, Bell PR, Thompson MM. Localization of matrix metalloproteinase 2 within the aneurysmal and normal aortic wall. BrJ Surg. 2000;87:1391-1400.

22. Fontaine V, Jacob MP, Houard $\mathrm{X}$ et al. Involvement of the mural thrombus as a site of protease release and activation in human aortic aneurysms. Am J Pathol. 2002;161:1701-1710.

23. Kuwahara F, Kai H, Tokuda $\mathrm{K}$ et al. Hypoxia-inducible factor-1alpha/vascular endothelial growth factor pathway for adventitial vasa vasorum formation in hypertensive rat aorta. Hypertension. 2002;39:46-50.

24. Stenbaek J, Kalin B, Swedenborg J. Growth of thrombus may be a better predictor of rupture than diameter in patients with abdominal aortic aneurysms. Eur J Vasc Endovasc Surg. 2000;20:466-469.

25. Hagisawa K, Nishioka T, Suzuki R et al. Enhancement of ultrasonic thrombus imaging using novel liposomal bubbles targeting activated platelet glycoprotein IIb/IIIa complex-in vitro and in vivo study. Int J Cardiol. 2011;152:202-206.

26. Pearlman JD. Medical imaging techniques in the evaluation of strategies for therapeutic angiogenesis. Curr Pharm Des. 2002;8:1467-1496.
27. Spadaccio C, Di DF, Perluigi M et al. Serum proteomics in patients with diagnosis of abdominal aortic aneurysm. Cardiovasc Pathol. 2012;21:283-290.

28. Wolski A, Chibowski D, Michalak J, Siezieniewska Z. Histoclinic of the abdominal aortic aneurysms. Ann Univ M Curie Sklod Med. 2001;56:171-176.

29. Vorp DA, Lee PC, Wang DH et al. Association of intraluminal thrombus in abdominal aortic aneurysm with local hypoxia and wall weakening. J Vasc Surg. 2001;34:291-299 .

30. Reeps C, Essler M, Pelisek J et al. Increased 18F-fluorodeoxyglucose uptake in abdominal aortic aneurysms in positron emission/computed tomography is associated with inflammation, aortic wall instability, and acute symptoms. J Vasc Surg. 2008;48:417-423.

31. Chan WL, Pejnovic N, Liew TV, Hamilton H. Predominance of Th2 response in human abdominal aortic aneurysm: mistaken identity for IL-4-producing NK and NKT cells? Cell Immunol. 2005;233:109-114.

32. Houard X, Ollivier V, Louedec L, Michel JB, Bäck M. Differential inflammatory activity across human abdominal aortic aneurysms reveals neutrophil-derived leukotriene B4 as a major chemotactic factor released from the intraluminal thrombus. FASEB J. 2009;23:1376-1383.

33. Bluestein D, Niu L, Schoephoerster RT, Dewanjee MK. Steady flow in an aneurysm model: correlation between fluid dynamics and blood platelet deposition. $J$ Biomech Eng. 1996;118:280-286.

34. Cronenwett JL. Variables that affect the expansion rate and rupture of abdominal aortic aneurysms. Ann N Y Acad Sci. 1996;800:56-67.

35. Nakahashi TK. Flow loading induces macrophage antioxidative gene expression in experimental aneurysms. Arterioscler Thromb Vasc Biol. 2002;22:2017-2022.

36. Wiernicki I, Stachowska E, Safranow K et al. Enhanced matrix-degrading proteolytic activity within the thin thrombuscovered wall of human abdominal aortic aneurysms. Atherosclerosis. 2010;212:161-165.

37. Michel JB, Martin-Ventura JL, Egido J et al. Novel aspects of the pathogenesis of aneurysms of the abdominal aorta in humans. Cardiovasc Res. 2011;90:18-27.

38. Mayranpaa MI, Trosien JA, Fontaine V et al. Mast cells associate with neovessels in the media and adventitia of abdominal aortic aneurysms. J Vasc Surg. 2009;50:388-295.

39. McDonald DM, Choyke PL. Imaging of angiogenesis: from microscope to clinic. Nat Med. 2003;9:713-725.

40. Ocak I, Baluk P, Barrett T, McDonald DM, Choyke P. The biologic basis of in vivo angiogenesis imaging. Front Biosci. 2007;12:3601-3616. 